\title{
Analysis of Cloud Security and Internet of Things
}

\author{
Peng Qian'; $\quad$ Yaowei Ding ${ }^{2}$ \\ ${ }^{1}$ Anhui San lian University, Hefei 230601, China \\ ${ }^{2}$ LCFC (Hefei) Electronics Technology Co.Ltd.230601, China
}

\begin{abstract}
With the advent of computer network, network security has been the core of information technology. We put forward a new concept after cloud calculating, that is, cloud security. Up to now, network security and cloud security have no difference essentially. In this paper, the advantages of cloud security and technology are described, along with the introduction of the hierarchical structure and application and analysis of the impact of cloud security on the Internet of things.
\end{abstract}

Keyword: Network security; Cloud Security; Internet of things

\section{NETWORK SECURITY}

With the continuous progress of science technology, the development of computer and network has a qualitative leap. The requirement that human activities rely on the computer network is increasingly strict. The most important thing is the network security. Generally speaking, network security includes two aspects, namely, internal and external factors.

\subsection{Internal and external factors}

\subsubsection{Internal factors}

Internal factors caused by the network security are mainly unit or the user itself, according to the actual investigation, the risk of the vast majority of network security comes from individuals. Main problems are as follows,

- Privilege control is not clear;

- Using pirated software or unknown mobile devices;

- The node information is asymmetric and problems that are not dealt with timely by users;

- Technical level is low and operation is mistaken;

- Playing a joke. Expressing their anger and Launch an attack internally

In light of the above phenomena, in order to remove the internal security factors from the network .therefore, we must take two aspects into account .such as technology and management ${ }^{[1]}$.

\subsubsection{External factor}

In addition to the internal factors,. Meanwhile, the security threats are also caused by external factors, it usually are divided into two kinds. One is active situation, and the other is passive situation.

(1) Active attacks: It is destructive and blindly purposeful , which tampers and fabricates data .Even worse, it leads to a large area of network paralysis. Meantime, it causes an immeasurable loss to the user. 
(2) Passive attack, compared with the former, its destructive power sometimes is not strong, and sometimes weak. Purpose is not clear. Mainly from the user "over the wall", browse the garbage sites, illegal access to information.

For external factors, we should do: one is to Supplement and upgrade system vulnerabilities. At the same time, install protection software and anti-virus software; another is to legitimate Internet access and browse regular web site.

\subsection{Cloud Security}

We are now talking about cloud security that is put forward by Chinese enterprises. In essence, there is no huge difference between network security and cloud security. The reason is that cloud security still adopts traditional solutions, While, it has slightly improved in terms of technology. The Internet of things is the extension and expansion of the computer network, which is in the form of exchange of one commodity for another to work on data exchange and network transmission. Therefore, the internet of things covers the field that cannot be separated from the cloud security.

Cloud security anti-virus method and traditional anti-virus methods differ in using thinking mode of the internet. All kinds of virus code are obtained by way of network technology, then the characteristics of virus are detected by artificial intelligence + mode, then the solution is sent to every client .Finally cloud security comprises a large network virus protection. For example, firstly one of the client terminals is attacked by virus, then cloud security utilizes the virus code that has gathered to check so that the user avoid being invaded. It is reported that the cloud security can support an average of 5 billion 500 million per day Click query, and analyse 250 million samples collected every day. The database can achieve the first hit rate of 99\%. With the help of cloud security, viruses blocked reach up to 10 million times ${ }^{[2]}$.

In addition, cloud security technology does not occupy the user's memory, and the virus has been intercepted before the invasion. The traditional anti-virus software needs to occupy a large number of users of memory, and some viruses that need upgrading can work.

\section{$2 S$ HIERARCHY AND APPLICATION OF THE INTERNET OF THINGS}

\subsection{What is the Internet of things}

The concept of Internet of things was first proposed by Professor Ash-ton Kevin of Massachusetts Institute of Technology in 1999. By way of the radio frequency identification (RFID) (RFID+ Internet), infrared sensors, GPS, laser scanners, gas sensors and other sensing devices, Anything are connected with the Internet, information exchange and communication According to the agreed protocol to realize intelligent identification, positioning, tracking, monitoring and management.

\subsection{Hierarchy and application of the Internet of things}

In October 30, 2016, the World internet of thing Expo held in Wu xi city, Jiangsu province, China, it can be learned from the "2015-2016 China networking development annual report". Global networking market invested \$7 trillion in 2015. Successful reasons benefit from the cloud security, cloud computing, wireless network technology and the enhancement of Internet equipment. In addition, the global interconnection device is growing exponentially, in 2015, about 4 billion 88 million units [3]. 
At present, the Internet of things in China has formed a relatively complete industrial chain, the structure of the Internet of things is divided into three layers, that is, the perception layer, network layer, and application layer. The structure of the model is as shown in figure 1. In addition, the three levels in the market has a large scale, its application is very wide, including: industrial, agricultural, business industry as well as medicine, transportation, construction, military, environment and so on. The specific case study, such sa: ZigBee wireless technology controlling the street lamp, access control system of AI , dynamic image that is transmitted by mobile phone, and other applications.

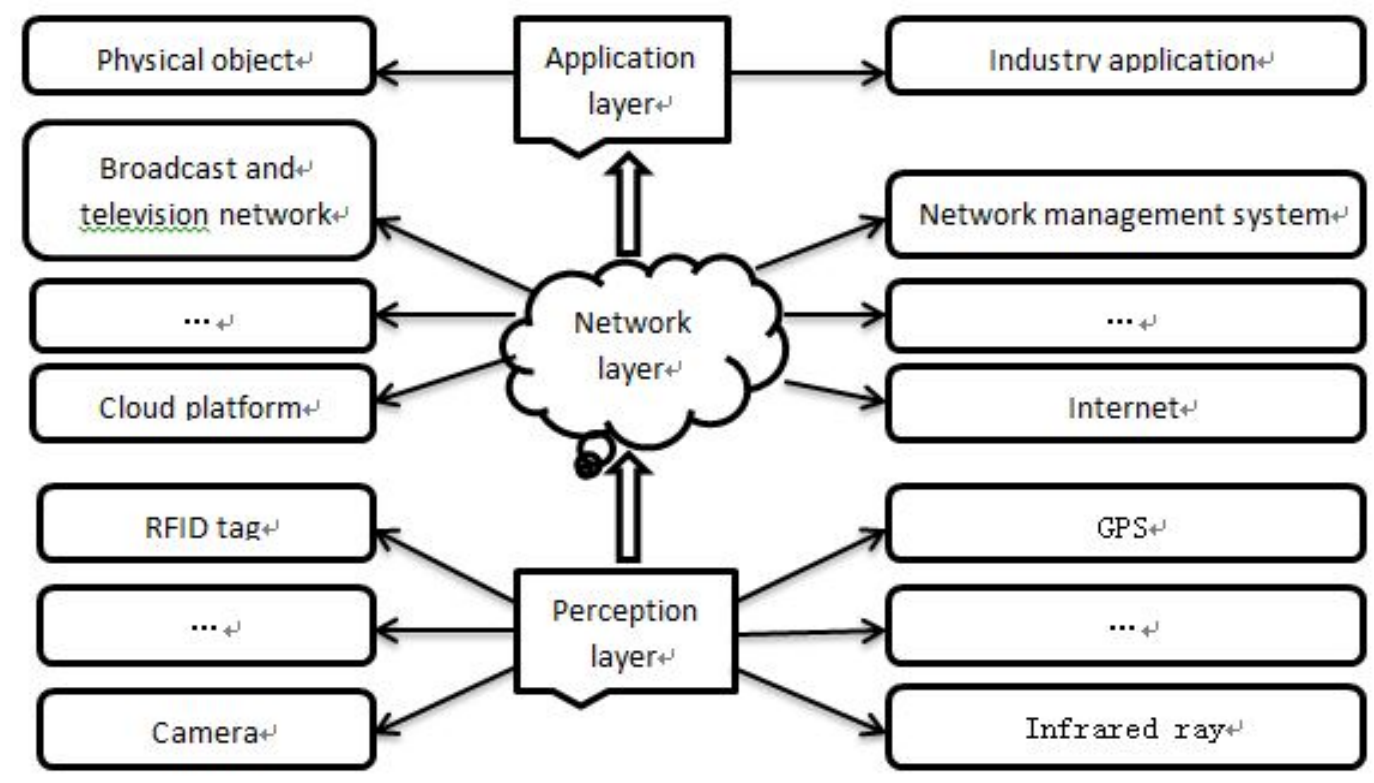

Fig. 1 hierarchy of Internet of things

\section{THE INFLUENCE OF CLOUD SECURITY ON INTERNET OF THINGS}

\subsection{Relationship between cloud security and Internet of things}

The core issue of the Internet of things is the data security and the right to control the data, cloud security is an important guarantee for the Internet of things. The realization of the Internet of things also requires a lot of other key technologies to support [4], such as radio frequency identification technology, embedded system technology, wireless network technology, etc. But these should take fully advantages of their functions. Obviously, cloud security is extremely important. For example, cloud monitoring of can transfer real-time picture to the user's mobile phone via WIFI. In this process, it is inevitable that criminals intercepted information for criminal purposes. Therefore, the development of the Internet of things cannot be separated from the cloud security, cloud security is to the internet of things what protector is to protected person.

\subsection{The advantages of cloud security for the Internet of things}

\subsubsection{Reasonable utilization of resources, cost saving, convenient use of users}

In the daily life, the application of the Internet of things will inevitably encounter some virus invasion, there are benign and malignant. Benign virus has little influence on the internet of things, 
While, the malignant virus causes the trouble or the economic loss to users. At present, users basically installed anti-virus software, and some also install different anti-virus software for different purposes. Even so, computer is likely to be invaded by the virus, or even because system resources occupied that leads to speed down the computer's running speed or computer crash. However, our cloud security is to monitor a large number of users and has access to resources of the latest virus, then analyses and processes by the server, finally sends the solutions to each user terminal so that the entire Internet can turn into super anti-virus software to solve these problems.

\subsubsection{Anti-virus and defence at any time}

As is known to all, whether anti-virus software is helpful mainly depends on its sensitivity to virus detection. The sensitivity of the traditional anti-virus software antivirus is high on condition that the virus is invading or invasion ends. Finally it begins detecting and blocking. Using this way will be a waste of time. Meanwhile, it cannot guarantee the safety of data. The emergence of cloud security technology just makes up for the flaw of the vulnerability. Before the virus invades the user terminal, it has been cleared in the cloud, and the detection rate is very high. Compared with the traditional anti-virus software, cloud security has an absolute advantage.

\subsubsection{Co-share Resource}

When the first user terminal is invaded by the virus, we should make the use of cloud security technology to deal with immediately. In addition, with the help of network, cloud security constantly update virus database in the cloud, so that other users will be free from attacks. The more network user terminals are, the richer cloud database is. Meantime it speeds up updating data. Realize the goal of co-sharing resources and provide the shadow to the internet of the things.

In summary, it goes without saying that cloud security exerts great influence on the Internet of things. With the fast growth and changing innovation of Global network, application-oriented innovation is the core of the development of things. To a large extent, it improves the work efficiency and gains economic benefits. Finally it reduces operating costs, Cloud security will be indispensable part of our daily life.

\section{Literature References}

[1]ZhaiZhong. The introduction of computer science[M], Tsinghua university Press, March , 2014 , the fourth edition

[2Baidu Encyclopedia http://baike. baidu. com

[3] The world of the Internet of

Things http://news. rfidworld. com. cn/2016 11/23505db49aa657ae. htm1

[4]liu Jing. Gu Jia Qiang. The Status quo of Internet of Things and Development Strategy in China, Enterprise Economy, 2013, the fourth edition ( Total $392^{\text {nd }}$ phase ) 114-115 